\title{
A Soft X-ray Polarimeter Designed for Broad-band X-ray Telescopes
}

\author{
Herman L. Marshall ${ }^{a}$ \\ ${ }^{a}$ MIT Kavli Institute, Cambridge, MA, USA
}

\begin{abstract}
A novel approach for measuring linear X-ray polarization over a broad-band using conventional imaging optics and cameras is described. A new type of high efficiency grating, called the critical angle transmission grating is used to disperse soft X-rays radially from the telescope axis. A set of multilayer-coated paraboloids re-image the dispersed X-rays to rings in the focal plane. The intensity variation around these rings is measured to determine three Stokes parameters: I, Q, and U. By laterally grading the multilayer optics and matching the dispersion of the gratings, one may take advantage of high multilayer reflectivities and achieve modulation factors over $50 \%$ over the entire 0.2 to $0.8 \mathrm{keV}$ band. A sample design is shown that could be used with the Constellation-X optics.
\end{abstract}

Keywords: X-ray, polarimeter, astronomy, multilayer, mirror, grating

\section{INTRODUCTION}

Although polarization is a fundamental property of electromagnetic radiation, there have been no astronomical measurements of polarization in the X-ray band in over $30 \mathrm{yr}$. There has been no lacking for workable concepts, including simple designs such as the Polarimeter for Low Energy X-ray Astrophysical Sources (PLEXAS) that was proposed by Marshall et al. to use multilayer-coated mirrors tuned to $0.25 \mathrm{keV}$ as Bragg reflectors. ${ }^{1}$ An excellent review of the history and prospects for astronomical polarimetry in the $0.1-10 \mathrm{keV}$ band is presented by Weisskopf et al. ${ }^{2}$ Weisskopf et al. rightly argue that the PLEXAS design has a narrow bandpass, reducing its attractiveness for astrophysical observations because one expects polarization to be energy dependent, so a wide bandpass is desired.

Here, I outline a new polarimeter design that can take advantage of broad-band X-ray optics by dispersing the incoming X-rays onto a multilayer-coated secondary. The graze angles off of the secondary are large, of order $30-40^{\circ}$, and the polarization is determined in aggregate by measuring intensity around rings in the focal plane. Compared to the PLEXAS design, the bandpass increases from about $20 \mathrm{eV}$ to over $500 \mathrm{eV}$. The basic approach is very flexible. For the sake of demonstration, a configuration is designed for the Constellation-X mission. A similar design could be used for the XEUS mission or be designed for a small explorer.

\section{PRIMARY MIRROR AND GRATINGS}

The approach to this polarimeter design was inspired by a new blazed transmission grating design, called the Critical Angle Transmission (CAT) grating, ${ }^{3}$ and the corresponding application to the Constellation-X (Con-X) mission in the design of a transmission grating spectrometer. ${ }^{4}$ For details of the Con-X mission, see the Con-X web site at http://constellation.gsfc.nasa.gov/. This type of grating gives very high efficiency in first and higher orders. With such high efficiency, a dispersed spectrum could be matched to a multilayer-coated secondary mirror that would then perform the function of a polarizer.

We assume that the primary mirror system is capable of focussing X-rays over the 0.1 to $1.0 \mathrm{keV}$ band. For Con-X, the baseline optics are four assemblies consisting of $n$ conical shells with an outer radius $r_{1}=0.65 \mathrm{~m}$ and an inner radius $r_{n}=0.15 \mathrm{~m}$. The effective area goal is $3.8 \mathrm{~m}^{2}$ using 4 independent mirror assemblies. The focal length $F=10.0 \mathrm{~m}$. The mirror resolution goal is $\delta \theta=5^{\prime \prime}$, while the baseline is $15^{\prime \prime}$.

Further author information: E-mail: hermanm@space.mit.edu, Telephone: 16172538573 


\section{MULTI-DETECTOR APPROACH}

One could convert the CAT-based spectrometer to a polarimeter very simply by adding multilayer-coated flats at the current locations of the readout CCD arrays to reflect the X-rays by $90^{\circ}$. The detectors would be repositioned and rotated $90^{\circ}$ to receive the reflected X-rays, facing these flats. Fig. 1 shows how the focal plane might look. The multilayer spacing, $d$, would vary laterally (along the dispersion) in order to provide optimal reflection at a graze angle $\theta=45^{\circ}$. Such optimization is obtained by equating the wavelength from the linearized grating equation, $m \lambda=P x / D_{R}$, to that of a multilayer, $\lambda=2 d \sin \theta$, giving

$$
d=\frac{P x}{2^{1 / 2} m D_{R}},
$$

where $x$ is the distance along the dispersion from 0th order, $D_{R}$ is the Rowland diameter (the distance between the gratings and the detector plane), $m$ is the grating order, and $P$ is the period of the gratings. Thus, the multilayer period varies linearly with $x$, providing high reflectivity in a narrow bandpass at large graze angles. For a given order, the spectrometer's gratings direct a narrow bandpass to any given position on the flat, so the bandpass of the multilayer can be similarly narrow. The bandpass of the multilayer decreases approximately as $1 / N$ for a large number of layers, $N$, so if the spectrometer has a resolution better than 100 , say, then $N \gtrsim 100$. Large $N$ also improves the multilayer reflectivity.

At Brewster's angle, $\theta=45^{\circ}$, reflectivity is minimized when the $E$-vector is in the plane containing the incident ray and the surface normal ( $p$-polarized) and maximized when the $E$-vector is in the surface plane (s-polarized). The polarization position angle (PA) is the average orientation in sky coordinates of the $E$-vector for the incoming X-rays. Sampling at least 3 PAs is required in order to measure three Stokes parameters (I, Q, $\mathrm{U})$ uniquely, so one would require at least three separate detector systems with accompanying multilayer-coated flats. This design is more costly and heavy than the original design by Flanagan et al. ${ }^{4}$ due to the need for a third detector system. Another problem is that $m$ has to be chosen in order to set the multilayer spacing, reducing efficiency in high orders, which are critical to achieving high throughput at short wavelengths. ${ }^{4}$ For these reasons, the rest of this paper will focus on a design that requires only one detector to measure the polarized signal and sets $m=1$.

\section{SINGLE DETECTOR SYSTEM}

If there is to be only one detector that is oriented perpendicular to the telescope axis, then the gratings can be designed to disperse the focussed X-rays away from the telescope axis and a set of multilayer-coated mirrors would redirect the light to the focal plane. This design is illustrated schematically in Fig. 2. In this case, the gratings are arranged to disperse radially from the optical axis, so that wavelength increases approximately linearly with distance $x$ from the optical axis.

\subsection{Disperser}

Transmission gratings are used to disperse the X-rays. The main requirements are to obtain a spectral resolution $R \equiv E / \delta E \gtrsim N \sim 100$ and to have high efficiency. The medium energy gratings (MEGs) of the Chandra X-ray Observatory High Energy Transmission Grating Spectrometer ${ }^{5}$ could be used, for example. The MEGs have a polyimide supporting membrane that reduces throughput between 0.3 and $0.5 \mathrm{keV}$, so a grating like that used for the Chandra Low Energy Transmission Grating Spectrometer ${ }^{6}$ (LETGS) might be more appropriate. However, the peak efficiency in +1 order is about $10 \%$ for these gratings, while that of the blazed CAT gratings can reach $50 \%{ }^{3}$ The CAT grating design used in this study has $P=100 \mathrm{~nm}$, bars that are $3.5 \mu$ tall, $15 \mathrm{~nm}$ wide, and tilted at $0.95^{\circ}$ to the vertical. The predicted first order efficiency (R. Heilmann, private communication), shown in Fig. 3. peaks at over 50\%. If placed just behind the Con-X mirrors, the CAT grating structure would have to be about $1.3 \mathrm{~m}$ across and the resolution at the focal plane would be $\gtrsim 1000$. This polarimeter design only requires $R \sim 100$, however, so the grating assembly can be placed about $1 \mathrm{~m}$ from the focal plane, reducing the size to about $130 \mathrm{~mm}$ diameter. The mass of the assembly is reduced by a factor of 100 . For this period and assuming $D_{R}=1 \mathrm{~m}$, the grating equation gives $x=\lambda$ for $x$ in $\mathrm{mm}$ and $\lambda$ in $\AA$. 

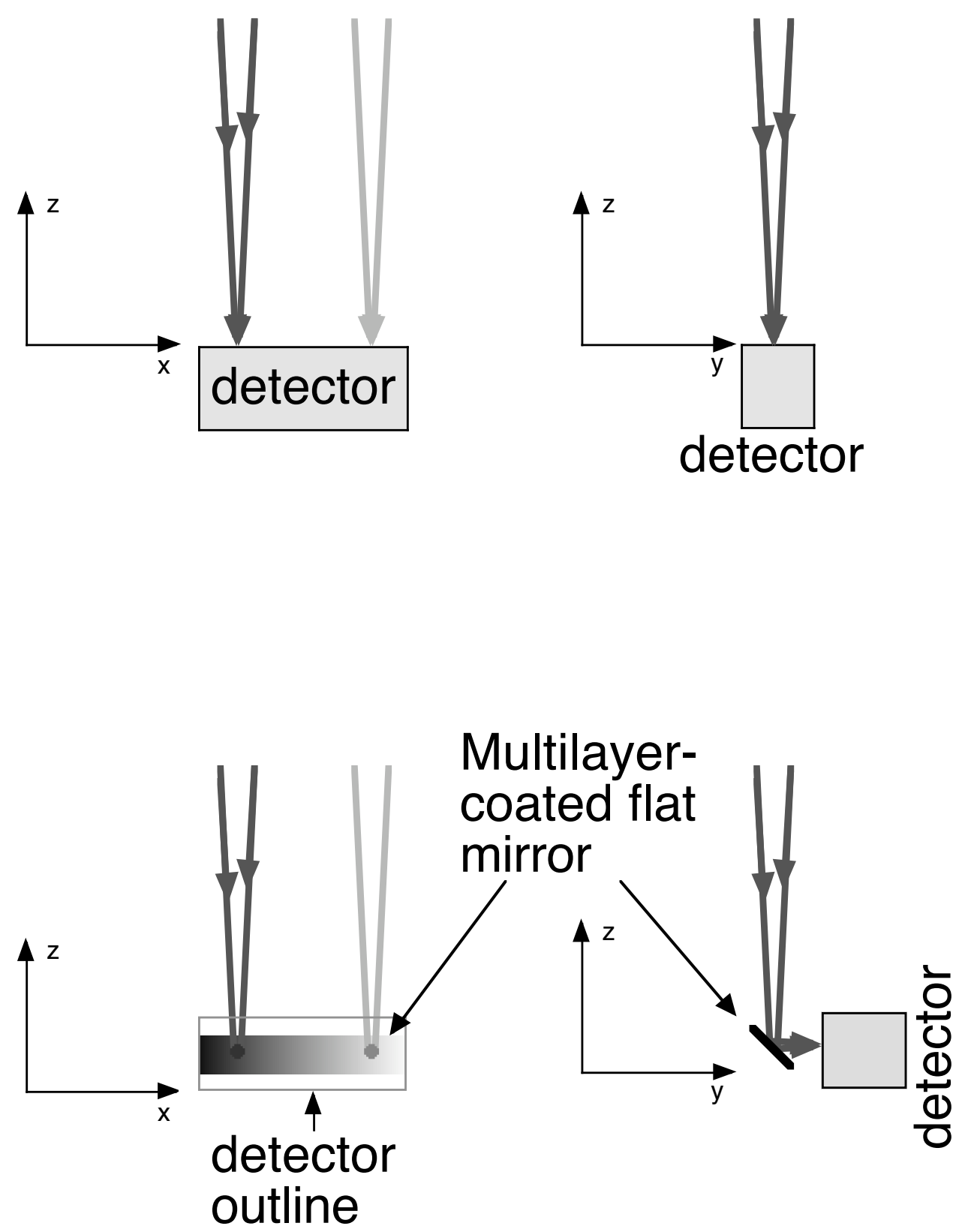

Figure 1. Top: Schematic of a single detector receiving dispersed X-rays from gratings behind the telescope, as in Constellation-X. Two views are shown, where the $z$ axis is along the telescope axis. The spectrometer dispersion axis is approximately parallel to the $x$ axis, along the surface of the detector (which is segmented to follow the Rowland circle). The cross-dispersion direction is parallel to the $y$ axis. Two different wavelengths of X-rays arrive at the focal plane, differing in $x$ according to the grating dispersion relation. Only one of these is shown in the right-hand view. Bottom: Same telescope and grating optics but now a polarizing flat has been added. The flat is placed at the Brewster angle $\left(45^{\circ}\right)$ to the incoming X-rays to null the reflectivity of $p$-polarized X-rays. The multilayer coating period varies with position along the $x$ axis according to Eq. (1), tuned to reflect short wavelengths at low $x$ and long wavelengths at large $x$. The detector is rotated $90^{\circ}$ about the $+x$ axis so that the focal plane is now approximately parallel to the $x-z$ plane. In the left-hand view, the detector would block the view of the multilayer-coated flat mirror, so only an outline of the detector is shown. Note that the flat and the detector are displaced in the $+z$ direction from the original focal plane. 


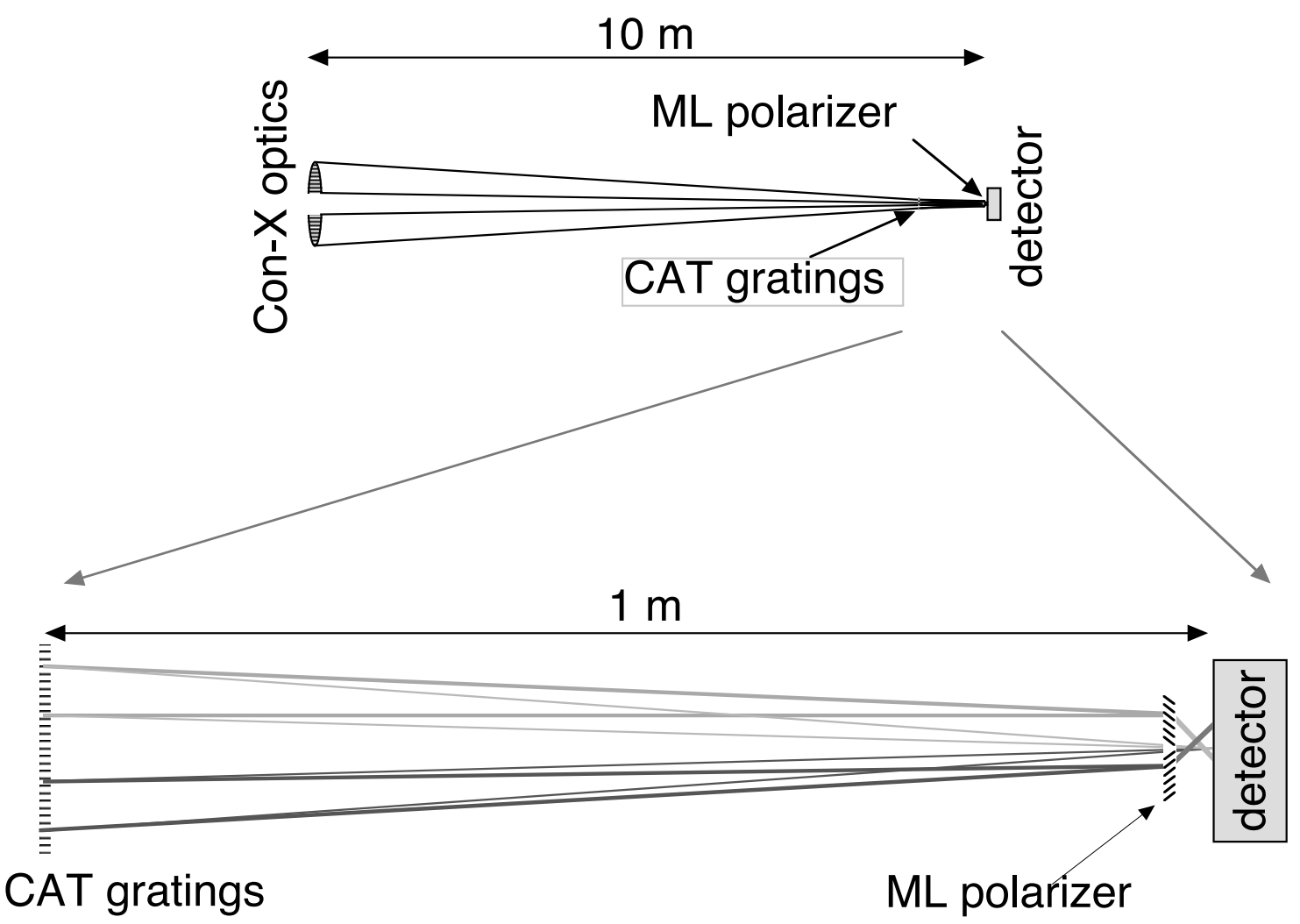

Figure 2. Top: The Constellation-X optical system with Critical Angle Transmission (CAT) gratings ${ }^{3}$ and multilayer (ML) polarizing optics added, approximately to scale (except for the detector size). The CAT gratings are $1 \mathrm{~m}$ from the focal plane and the multilayer polarizers are 15-60 mm from the detector. Bottom: Blowup of the polarizing optics. The CAT gratings disperse away from the telescope focus; 0th order light (thin lines) continue to the focus. Low energy (light gray) or high energy (dark gray) X-rays are dispersed so that wavelength increases from the optical axis. The polarizer reflects at large graze angles so that the intensity around the ring provides polarization information. 


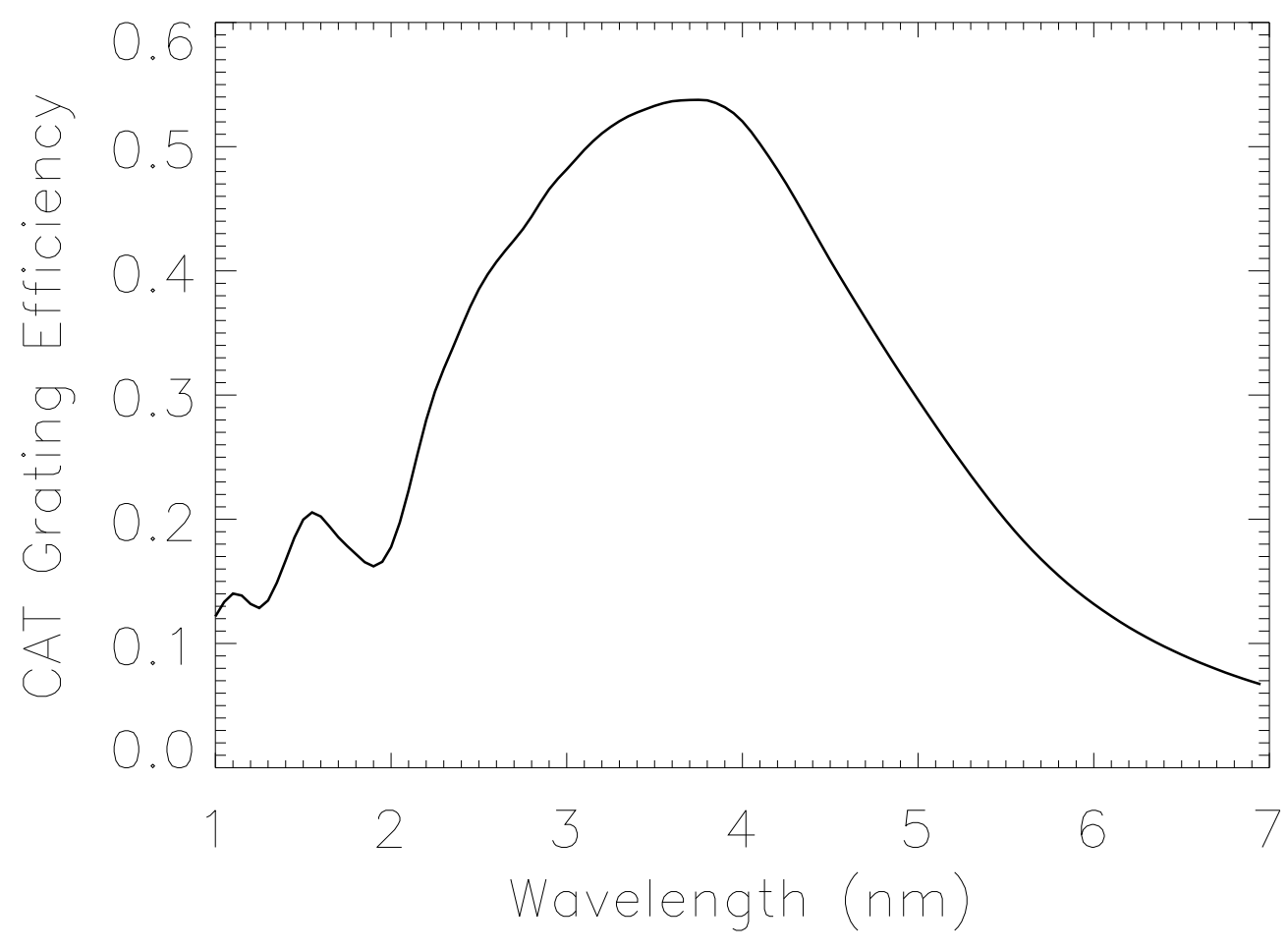

Figure 3. Computed efficiency of the CAT gratings ${ }^{3}$ in first order used in this study (R. Heilmann, private communication). The CAT grating design has $P=100 \mathrm{~nm}$, bars that are $3.5 \mu$ tall, $15 \mathrm{~nm}$ wide, and tilted at $0.95^{\circ}$ to the vertical. The efficiency peaks over $50 \%$.

\subsection{Multilayer Polarizer}

The final optical element is a set of nested reflectors that are approximately paraboloidal. A cross section through the rings is shown in Fig. 4. The ring spacing was chosen to reflect the radially dispersed X-rays into a ring image. Each paraboloid has a different image ring size and reflects a different $1 \mathrm{~nm}$ bandpass in this design. Highest efficiency is achieved when a narrow bandpass from the gratings matches the narrow reflectivity response of the multilayer. The reflection angles of the paraboloids are set in order to obtain graze angles between 25 and $40^{\circ}$ from considerations of the modulation factor and from the requirement that any given paraboloid does not occult the light reflected off of another.

The images that might be produced are shown schematically in Fig. 5. In this figure, the outermost ring image is generated by the innermost paraboloid: the $1-2 \mathrm{~nm}$ wavelength range (0.62 to $1.24 \mathrm{keV})$. Conversely, the innermost ring image is formed by the most highly dispersed X-rays, with wavelengths between 6 and $7 \mathrm{~nm}$ $(0.18$ to $0.21 \mathrm{keV})$. The nested reflectors should be coated so as to reflect only those X-rays at the appropriate Bragg condition. The graze angles are shown in Fig. [6, along with the required multilayer period, $d$. Following Marshall et al., ${ }^{7}$ the intensity $I_{i}$ around ring image $i$ in the $\delta \lambda$ band about $\lambda_{i}$ varies with azimuthal angle $\phi$ as

$$
I_{i}(\phi)=I_{i}^{0}\left\{\frac{1}{2 \pi}+P_{i}^{0} M \cos \left(2\left[\phi-\varphi_{\mathrm{i}}\right]\right)\right\},
$$

where $I_{i}^{0}$ is directly related to the I Stokes parameter of the source for bandpass $i, P_{i}^{0}$ is the average polarization fraction across the wavelength band, $\varphi_{i}$ is the phase determined by the orientation of average polarization $E$ vector on the sky (i.e., the polarization PA), and $M_{i}$ is the average system modulation factor in bandpass $i$. 


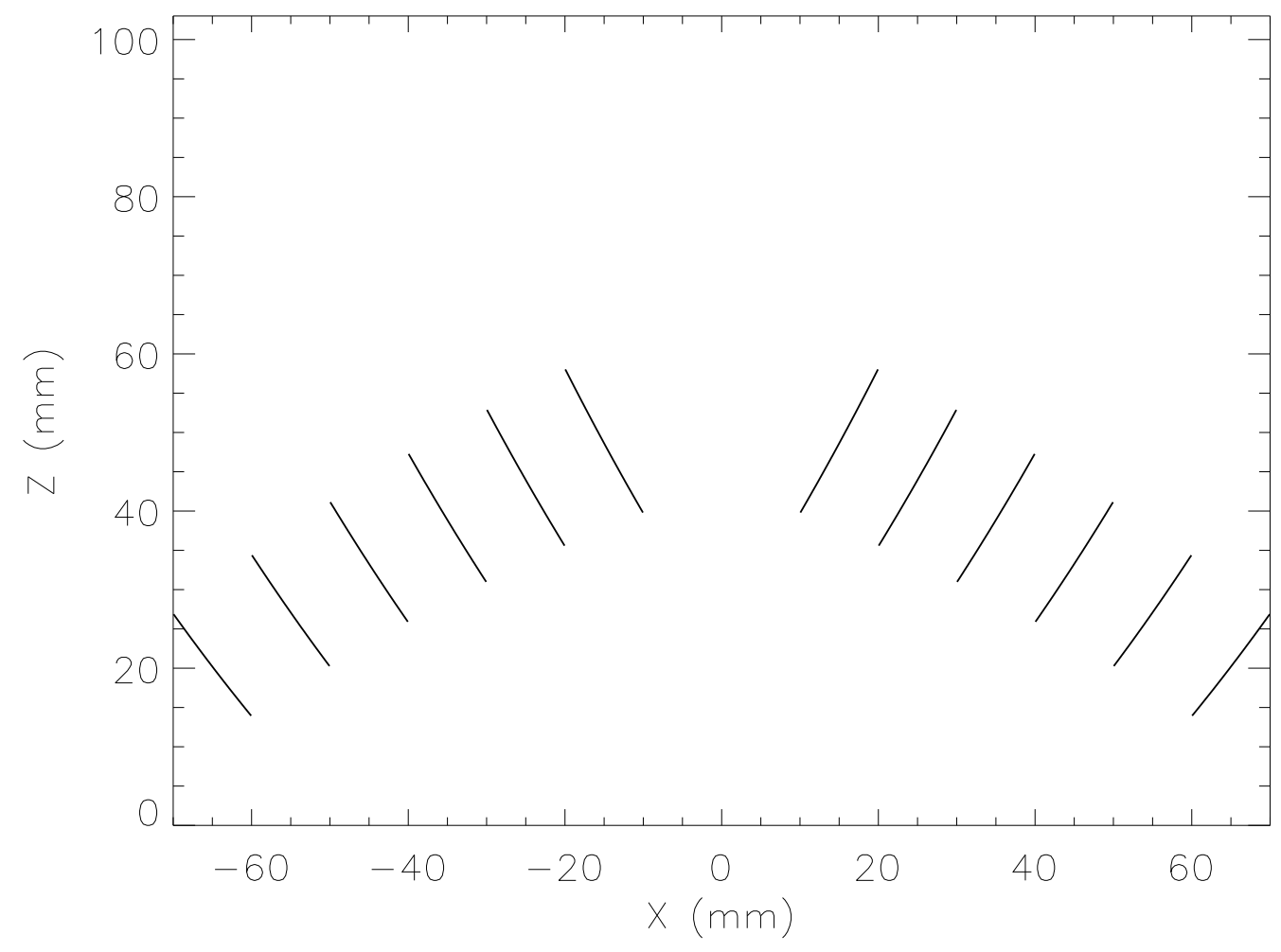

Figure 4. Cross section of the nested paraboloids that are multilayer coated to reflect with high efficiency in specific wavelength bands. The paraboloids redirect X-rays from the gratings into narrow rings in the focal plane (see Fig. 5 ). Each surface has a laterally graded multilayer that is tuned to the wavelength arriving there from the grating. Other configurations were studied and are not shown. The innermost ring intercepts X-rays with wavelengths between 1 and 2 $\mathrm{nm}$, the next ring reflects $2-3 \mathrm{~nm}$ X-rays, etc. 


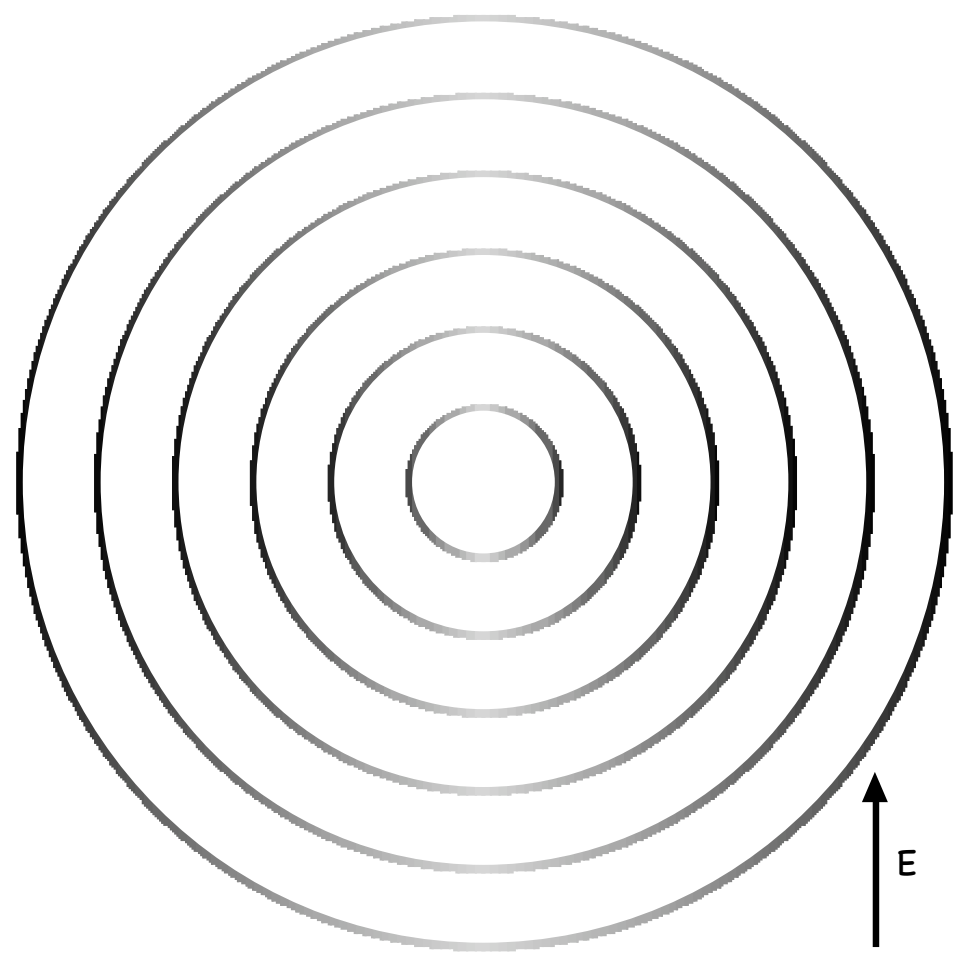

Figure 5. Ring images produced by the multilayer optics. Each ring corresponds to a different wavelength interval. The widths of the ring images are related to the mirror angular resolution and are generally $<10 \%$ of the distance between rings. The intensity around each ring varies as $\cos (2[\phi-\varphi])$, where $\phi$ is the azimuthal angle, and $\varphi$ determined by the $E$-vector of the polarized X-rays. Here, $E$ is parallel to the $y$ axis for each ring, so ring intensity is maximized along the $x$ axis. In general, the minima of each ring may differ, indicating a wavelength-dependent polarization position angle. The relative intensities of the rings depends on the source spectrum and the system throughput; here, a flat spectrum is assumed.

At any given wavelength, $M=\frac{R_{s}-R_{p}}{R_{s}+R_{p}}$, where $R_{s}$ and $R_{p}$ are the reflectivities for the $s$ and $p$ polarizations, respectively. The Stokes $\mathrm{Q}$ and $\mathrm{U}$ parameters can be derived from $P_{0}$ and $\varphi$ as a function of wavelength.

\subsection{System Design Issues}

The widths of the rings would be limited by the optical system, including the multilayer-coated optics. For Con-X with $5^{\prime \prime}$ primary optics, the rings would be at least $0.25 \mathrm{~mm}$ wide, so there is some room in the focal plane between ring images for background measurement and additional sources in the field. The field of view would be limited by the condition that the change of the graze angle on the paraboloids keep the X-ray within the spectral response band of the $N$-layer coating, $R \sim N \sim 100$. Differentiating the condition on the multilayer central wavelength, we find $2 d \cos \theta \delta \theta=\delta \lambda$, giving $\delta \theta=\tan \theta \delta \lambda / \lambda=\tan \theta / R \sim 0.01 \tan \theta$. For $\theta$ given in Fig. 6, $\delta \theta=0.3-0.5^{\circ}-$ much larger than the distance between the rings, which corresponds to $3.5^{\prime}$.

More important is the spread of the focussed X-rays as they exit the primary mirror assembly. In this design, where only one order is used, the polarizer receives X-rays over an angular range $\frac{r_{1}-r_{n}}{F}=0.05$ rad, or about $2.9^{\circ}$. The spread should be narrower than $\frac{\tan \theta}{N}$, so the innermost useful radius at the telescope aperture cannot be as small as $0.15 \mathrm{~m}$ as in the Con-X baseline. The gratings need not intercept the full incoming beam, however. The innermost radius corresponds to $r_{1}-\frac{F \tan \theta}{N}=0.51 \mathrm{~m}$ for an average graze angle of $35^{\circ}$. Limiting the beam this way cuts the effective area by a factor of 2.5. Alternatively, if the number of multilayers was reduced to $N=70$, say, there would be about a $35 \%$ gain in usable aperture, offset by a $\sim 30 \%$ loss of peak reflectivity. The multilayer reflectivity losses depend on wavelength, so a tradeoff study would be required. 


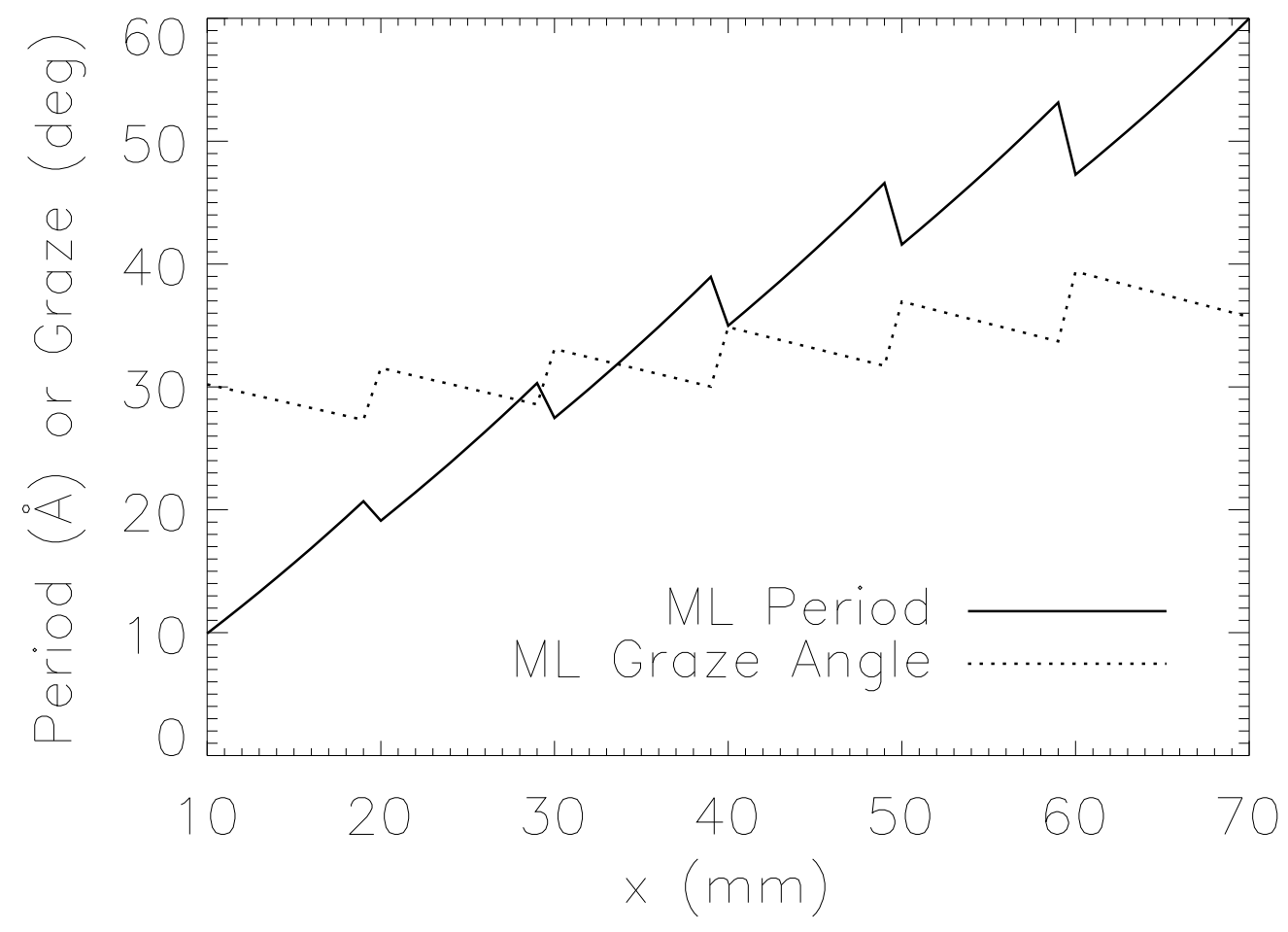

Figure 6. Variation of the multilayer period, ( $d$, solid line), and the graze angle ( $\theta$, dotted line) with X-ray wavelength. For the assumed grating period and placement of the grating from the focal plane, X-rays with wavelength $\lambda$ in $\AA$ disperse to distance $x=\lambda$, for $x$ in mm. The discontinuities result from segmenting the multilayer-coated polarizer.

The inner region of the focal plane is not used for this polarimeter design. This region is about $3.5^{\prime}$ in diameter, which satisfies the Con-X field of view requirement: $5^{\prime} \times 5^{\prime}$. This design could be used in conjunction with a high energy non-dispersive spectrometer because the gratings have a high 0th order efficiency, approaching $100 \%$ above $2 \mathrm{keV}$. Thus, low energy polarimetry would not interfere with high resolution spectroscopy, the primary objective of the Con-X mission, and the data would be obtained simultaneously.

\subsection{System Throughput and Sensitivity}

Using the Center for X-ray Optics web page (http://henke.lbl.gov/optical_constants/multi2.html), multilayer efficiencies were computed for a small range of materials for many of the relevant graze angles and corresponding $d$ values. For this work, $N$ was set to 100 and the interdiffusion thickness was set to $0.3 \mathrm{~nm}$, as has been achieved by several groups. ${ }^{8-10}$ Table 1 gives a sampling of the results, where $r$ is the peak reflectivity to unpolarized X-rays, and $\Gamma$ gives the fraction $d$ that is occupied by the first material given.

Assuming that this system is used in conjunction with one Con-X telescope (out of four), the geometric area in the $.2-1 \mathrm{keV}$ band is about $3300 \mathrm{~cm}^{2}$, after accounting for the factor of 2.5 loss described in the last section. For the purposes of this analysis, a CCD detector is assumed to have a thin Al overlayer to act as an optical blocking filter. The resulting effective area is shown in Fig. 7 along with the modulation factor, $M$.

The effective area estimate can be used to predict the minimum detectable polarization ${ }^{2}$ (MDP) for potential targets. As described by Marshall et al, ${ }^{1}$ extragalactic sources such as the BL Lac object PKS $2155-304$ are expected to be highly polarized in the X-ray band. In a 10,000 s observation of PKS 2155-304, this instrument could detect polarizations of 3-7\% in each of four bandpasses $1 \mathrm{~nm}$ wide from 2 to $6 \mathrm{~nm}(0.21$ to $0.62 \mathrm{keV})$. 
Table 1. Sample Multilayer Specifications

\begin{tabular}{|c|ccccccc|}
\hline $\begin{array}{c}\lambda \\
(\mathrm{nm})\end{array}$ & $\begin{array}{c}E \\
(\mathrm{keV})\end{array}$ & $\begin{array}{c}\theta \\
\left({ }^{\circ}\right)\end{array}$ & $\begin{array}{c}d \\
(\mathrm{~nm})\end{array}$ & $r$ & $M$ & Materials & $\Gamma$ \\
\hline 1.3 & 0.954 & 29.2 & 1.34 & 0.03 & 0.56 & $\mathrm{NaBr} / \mathrm{Ir}$ & 0.6 \\
1.7 & 0.729 & 28.0 & 1.82 & 0.08 & 0.49 & $\mathrm{NaF} / \mathrm{W}$ & 0.6 \\
2.0 & 0.620 & 31.5 & 1.92 & 0.10 & 0.62 & $\mathrm{NaF} / \mathrm{Ni}$ & 0.6 \\
2.5 & 0.496 & 29.9 & 2.53 & 0.14 & 0.53 & $\mathrm{SiO}_{2} / \mathrm{Cr}$ & 0.6 \\
3.2 & 0.387 & 32.4 & 2.99 & 0.27 & 0.53 & $\mathrm{Sc} / \mathrm{Cr}$ & 0.6 \\
3.8 & 0.326 & 30.4 & 3.81 & 0.19 & 0.48 & $\mathrm{Sc} / \mathrm{Cr}$ & 0.6 \\
4.0 & 0.310 & 34.9 & 3.54 & 0.15 & 0.67 & $\mathrm{Sc} / \mathrm{Cr}$ & 0.6 \\
4.4 & 0.282 & 33.5 & 4.05 & 0.14 & 0.53 & $\mathrm{Sc} / \mathrm{Cr}$ & 0.6 \\
4.5 & 0.276 & 33.1 & 4.16 & 0.29 & 0.56 & $\mathrm{C} / \mathrm{Cr}$ & 0.7 \\
4.9 & 0.253 & 31.7 & 4.66 & 0.26 & 0.54 & $\mathrm{C} / \mathrm{Cr}$ & 0.7 \\
5.0 & 0.248 & 36.9 & 4.16 & 0.20 & 0.78 & $\mathrm{C} / \mathrm{Cr}$ & 0.7 \\
6.0 & 0.207 & 39.4 & 4.83 & 0.16 & 0.88 & $\mathrm{C} / \mathrm{Cr}$ & 0.7 \\
7.0 & 0.177 & 35.7 & 6.00 & 0.15 & 0.74 & $\mathrm{C} / \mathrm{Cr}$ & 0.7 \\
\hline
\end{tabular}

In addition, isolated neutron stars such as RX J0720.4-3125 are expected to polarized due to effects of photon propagation in strong magnetic fields. ${ }^{2}$ In this case, it is worthwhile to obtain phase-resolved polarization data. In a 200,000 s observation with this instrument, one could reach MDPs of 5-8\% over the $2-5 \mathrm{~nm}$ band (in $1 \mathrm{~nm}$ wide bands) for each of 10 phase bins.

\subsection{Design Variations}

There is substantial flexibility to this design. For example, one may sacrifice imaging if, say, only bright point sources in uncrowded regions were to be observed. In this case, the polarizer needn't focus the dispersed X-rays but merely needs to redirect the X-rays to the detector. The polarizer could then consist of conic sections (frustums), making them easier to manufacture and coat. If the multilayer coating requirements are relaxed, then one may be able to use unblazed gratings (such as used in the LETGS) and capture both +1 and -1 orders, doubling the system throughput at what might be a small loss in multilayer efficiency.

The grating period need not be as small as $100 \mathrm{~nm}$. Larger periods may be used but the structure would have to be correspondingly closer to the mirror, increasing the size and mass of the assembly. For example, gratings with $P=1000 \mathrm{~nm}$ could be placed right behind the Con-X optics at about $10 \mathrm{~m}$ from the focal plane. For reference, the LETGS gratings have $P=991 \mathrm{~nm} .{ }^{6}$ The efficiency of the system is poor above $0.8 \mathrm{keV}$, where efficient multilayer coatings have not yet been demonstrated. Thus, the mirror need not have substantial effective area above $1 \mathrm{keV}$, or one may place the gratings only on the telescope perimeter.

There are many combinations of elements and multilayer coating parameters that were not investigated for this preliminary study, so one might expect to improve upon the mulilayer-coating reflectivities. The number of multilayers was held fixed at 100 and no in-depth $d$ variations were considered, in the interest of simplicity. It may be possible to superpolish the substrates to obtain $0.1 \mathrm{~nm}$ roughness,${ }^{11}$ which would increase reflectivities significantly.

\section{ACKNOWLEDGMENTS}

This work was supported under contract. I thank Dr. Ralf Heilmann for calculations of the CAT grating efficiencies for this analysis.

\section{REFERENCES}

1. H. L. Marshall, S. S. Murray, J. H. Chappell, H. W. Schnopper, E. H. Silver, and M. C. Weisskopf, "Realistic, inexpensive, soft x-ray polarimeter and the potential scientific return," in Polarimetry in Astronomy. Edited 


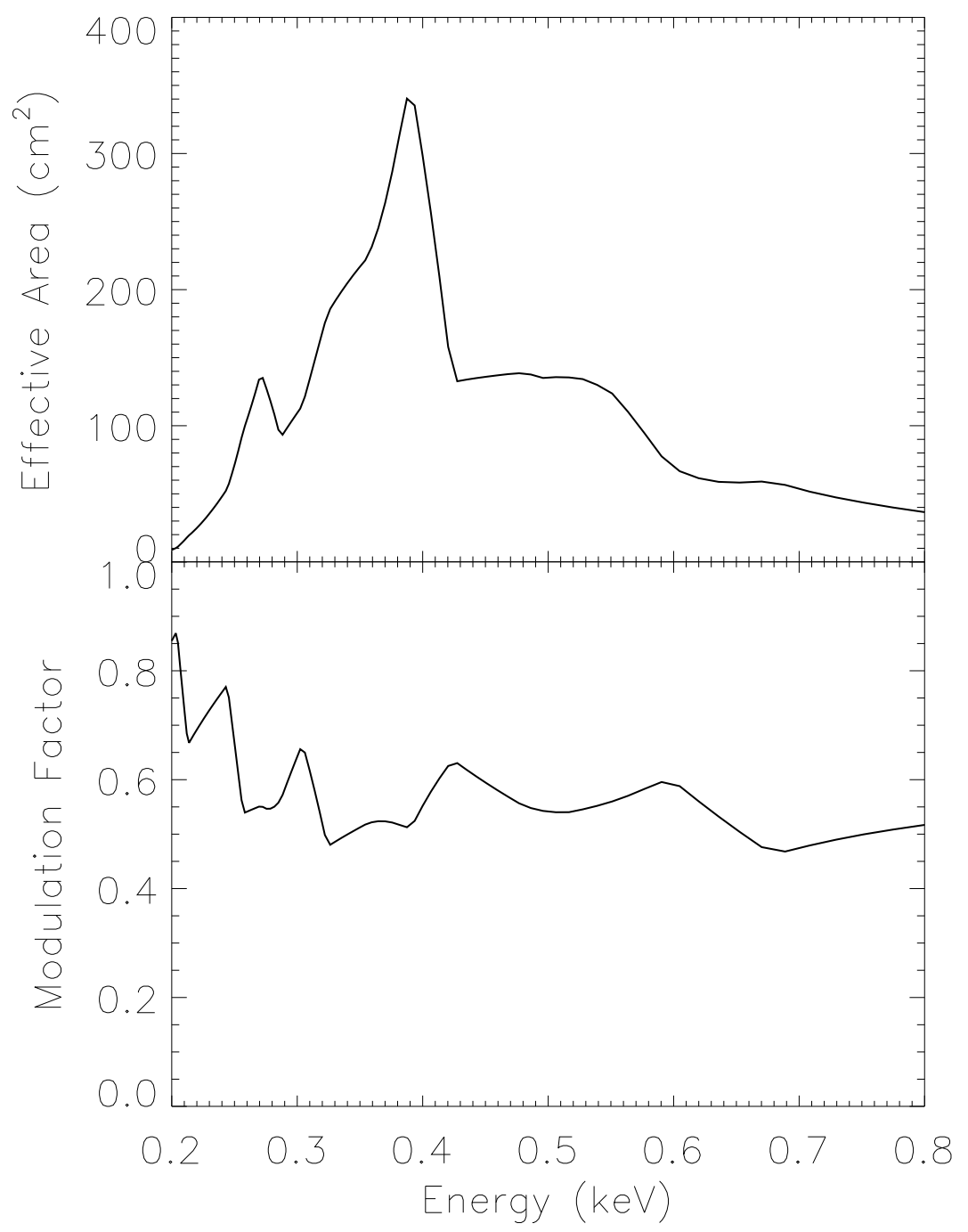

Figure 7. Effective area (top) and the polarization modulation factor (bottom) for a system where one Con-X mirror is used with a CCD detector and a thin optical blocking filter. An effective area of over $100 \mathrm{~cm}^{2}$ can be achieved over most of the $0.2-0.8 \mathrm{keV}$ bandpass.

by Silvano Fineschi. Proceedings of the SPIE, Volume 4843, pp. 360-371 (2003)., S. Fineschi, ed., Presented at the Society of Photo-Optical Instrumentation Engineers (SPIE) Conference 4843, pp. 360-371, Feb. 2003.

2. M. C. Weisskopf, R. F. Elsner, D. Hanna, V. M. Kaspi, S. L. O’Dell, G. G. Pavlov, and B. D. Ramsey, "The prospects for X-ray polarimetry and its potential use for understanding neutron stars," ArXiv Astrophysics e-prints, Nov. 2006.

3. R. K. Heilmann, M. Ahn, K. A. Flanagan, and M. L. Schattenburg, "Fabrication and charicterization of blazed transmission gratings for X-ray Spectroscopy," in Optics for EUV, X-Ray, and Gamma-Ray Astronomy. Edited by O'Dell, Stephen L.; Pareschi, Giovanni. Proceedings of the SPIE, Volume 6688, these proceedings., S. L. O'Dell and G. Pareschi, eds., Presented at the Society of Photo-Optical Instrumentation Engineers (SPIE) Conference 6688, 2007.

4. K. A. Flanagan, J. E. Davis, R. K. Heilmann, D. P. Huenemoerder, A. P. Levine, H. L. Marshall, G. Y. Prigozhin, G. R. Ricker, M. L. Schattenburg, N. S. Schulz, , and A. P. Rasmussen, "Spectrometer concept and 
design using a blazed transmission grating for x-ray astronomy," in Optics for EUV, X-Ray, and Gamma-Ray Astronomy. Edited by O'Dell, Stephen L.; Pareschi, Giovanni. Proceedings of the SPIE, Volume 6688, these proceedings., S. L. O'Dell and G. Pareschi, eds., Presented at the Society of Photo-Optical Instrumentation Engineers (SPIE) Conference 6688, 2007.

5. C. R. Canizares, J. E. Davis, D. Dewey, K. A. Flanagan, E. B. Galton, D. P. Huenemoerder, K. Ishibashi, T. H. Markert, H. L. Marshall, M. McGuirk, M. L. Schattenburg, N. S. Schulz, H. I. Smith, and M. Wise, "The Chandra High-Energy Transmission Grating: Design, Fabrication, Ground Calibration, and 5 Years in Flight," PASP 117, pp. 1144-1171, Oct. 2005.

6. A. C. Brinkman, C. J. T. Gunsing, J. S. Kaastra, R. L. J. van der Meer, R. Mewe, F. Paerels, A. J. J. Raassen, J. J. van Rooijen, H. Bräuninger, W. Burkert, V. Burwitz, G. Hartner, P. Predehl, J.-U. Ness, J. H. M. M. Schmitt, J. J. Drake, O. Johnson, M. Juda, V. Kashyap, S. S. Murray, D. Pease, P. Ratzlaff, and B. J. Wargelin, "First Light Measurements of Capella with the Low-Energy Transmission Grating Spectrometer aboard the Chandra X-Ray Observatory," ApJ 530, pp. L111-L114, Feb. 2000.

7. H. L. Marshall, C. M. Urry, R. M. Sambruna, and J. E. Pesce, "EUVE Observations of PKS 2155-304: Variability, Spectra, and a Polarization Measurement Attempt," ApJ 549, pp. 938-947, Mar. 2001.

8. D. L. Windt, S. Donguy, J. F. Seely, B. Kjornrattanawanich, E. M. Gullikson, C. C. Walton, L. Golub, and E. DeLuca, "EUV multilayers for solar physics," in Optics for EUV, X-Ray, and Gamma-Ray Astronomy. Edited by Citterio, Oberto; O'Dell, Stephen L. Proceedings of the SPIE, Volume 5168, pp. 1-11 (2004)., O. Citterio and S. L. O'Dell, eds., Presented at the Society of Photo-Optical Instrumentation Engineers (SPIE) Conference 5168, pp. 1-11, Feb. 2004.

9. H.-C. Mertins, F. Schäfers, H. Grimmer, D. Clemens, P. Böni, and M. Horisberger, "W C, W Ti, Ni Ti, and Ni V Multilayers for the Soft-X-ray Range: Experimental Investigation with Synchrotron Radiation," Appl.Optics 37, pp. 1873-1882, Apr. 1998.

10. C. Montcalm, P. A. Kearney, J. M. Slaughter, B. T. Sullivan, M. Chaker, H. Pépin, and C. M. Falco, "Survey of Ti-, B-, and Y-based soft x-ray -extreme ultraviolet multilayer mirrors for the 2- to 12-nm wavelength region," Appl.Optics 35, pp. 5134-5147, Sept. 1996.

11. U. Kleineberg, T. Westerwalbesloh, O. Wehmeyer, M. Sundermann, A. Brechling, U. Heinzmann, M. Haidl, and S. Muellender, "Bufferlayer and caplayer engineering of Mo/Si EUVL multilayer mirrors," in Proc. SPIE Vol. 4506, p. 113-120, Soft X-Ray and EUV Imaging Systems II, Daniel A. Tichenor; James A. Folta; Eds., D. A. Tichenor and J. A. Folta, eds., Presented at the Society of Photo-Optical Instrumentation Engineers (SPIE) Conference 4506, pp. 113-120, Dec. 2001. 